\title{
Influência do Controle Virológico e Terapia Antirretroviral na Força Muscular Respiratória em Sujeitos com HIV
}

\author{
Geovana de Almeida Righi ${ }^{1}$, Naiára Casarin ${ }^{2}$, Matheus Barros Moreira ${ }^{3}$, Gabriela Castro \\ Kuinchtner ${ }^{4}$, Tainara Tolves ${ }^{5}$, Luis Ulisses Signori ${ }^{6}$, Antônio Marcos Vargas da Silva ${ }^{7}$
}

\begin{abstract}
RESUMO
Objetivo: avaliar a influência do controle virológico e do uso de terapia antirretroviral (Tarv) sobre a força muscular respiratória em pacientes com Human Immunodeficiency Virus (HIV). Métodos: estudo transversal com 60 sujeitos com HIV, de ambos os sexos, em uso da terapia antirretroviral há pelo menos três meses ou sem uso. Os indivíduos foram subdivididos em três grupos: com Tarv e carga viral não detectável (GTCV-; $n=20$ ), com Tarv e carga viral detectável (GTCV+; $n=20$ ) e sem Tarv e carga viral detectável (GsTCV+; $\mathrm{n}=20$ ). A força muscular respiratória foi mensurada com um manovacuômetro digital, e os valores preditos das pressões respiratórias máximas calculados pela equação de Neder et al. (1999). Considerou-se fraqueza muscular inspiratória valores de pressão inspiratória máxima (PImáx) < $70 \%$ do predito. Resultados: a carga viral foi menor no GTCV+ do que no GsTCV+. A contagem de células T-CD4 foi maior no GTCV- do que nos demais. Os grupos GTCV+ $\left(60,5[37,1-70,5] \mathrm{cmH}_{2} \mathrm{O}\right)$ e GsTCV+ $\left(67,9[50,3-93] \mathrm{cmH}_{2} \mathrm{O}, \mathrm{p}<0,004\right)$ apresentaram redução da pressão expiratória máxima (PEmáx) na comparacão com o GTCV- $(100,2$ [71-121,9]cmH O), baseado no $\%$ do predito. Tanto o GTCV+ quanto o GsTCV+ mostraram valores de mediana que demonstram fraqueza muscular inspiratória. Conclusões: o grupo de pacientes em uso da Tarv e com carga viral não detectável apresentou maior força muscular expiratória e não foi classificado com fraqueza muscular inspiratória. Estes efeitos favoráveis da Tarv e da baixa carga viral podem implicar melhores desfechos funcionais, que devem ser testados em futuros trabalhos.

Palavras-chave: HIV. Testes de função respiratória. Terapia antirretroviral.

INFLUENCE OF VIROLOGICAL CONTROL AND ANTIRETROVIRAL THERAPY

ON RESPIRATORY MUSCLE STRENGHT IN SUBJECTS WITH HIV

ABSTRACT

Objective: to evaluate the influence of the viral control and the use of the antiretroviral therapy on the respiratory muscle strength in HIV patients. Methods: Cross-sectional study, 60 patients with HIV, both sexes, on antiretroviral therapy for at least three months or no use. They were subdivided in three groups: with ART and viral load not detectable (AGVL-; $n=20$ ); with ART and viral load detectable ( $A G V L+; n=20)$; without ART and viral load detectable ( $n A G V L+; n=20)$. The respiratory muscle strength was measured with a digital manovacuometer and the prediction values of the maximal respiratory pressure calculed using Neder et al., 1999 equation. We considered inspiratory muscle weakness values of maximal inspiratory pressure (MIP) $<70 \%$ of the predicted. Results: the viral load was lower in the AGVL+ than in nAGVL+, since the AGVL- showed viral load not detectable. The counting of T-CD4 was bigger in AGVL- than the others. The groups AGVL+ $\left(60.5\right.$ [37.1-70.5] $\left.\mathrm{cmH}_{2} \mathrm{O}\right)$ and $n A G V L+; n=20(67.9$ [50.3-93]cmH $0, p<0.004)$ presented reduction of maximal expiratory pressure (MEP) compared with AGVL- $\left(100,2\right.$ [71-121.9] $\left.\mathrm{cmH}_{2} \mathrm{O}\right)$, according to the predict percentage. The AGVL+ as well as the nAGVL+ has showed median values demonstrating inspiratory muscle weakness. Conclusions: The patients group using of ART and with load viral not detectable showed bigger expiratory muscle strength and was not classified with inspiratory muscle weakness. These favorable effects of the ART and of lower load viral may result in better functional outcomes which should be tested in future studies. Keywords: HIV. Pulmonary function tests. Antiretroviral therapy.
\end{abstract}

RECEBIDO EM: 16/10/2019

MODIFICAÇÕES SOLICITADAS EM: 26/12/2019

ACEITO EM: 5/3/2020

\footnotetext{
Fisioterapeuta pela Universidade Federal de Santa Maria (UFSM). Mestrado em Reabilitação Funcional pela UFSM. http://lattes.cnpq.br/8263068007136620. https://orcid.org/0000-0002-9842-4930. righigeovana@gmail.com

2 Fisioterapeuta pelo Centro Universitário Franciscano (Unifra). Mestrado em Ciências da Saúde pela UFSM. http://lattes.cnpq.br/2645518446782592. http:// orcid.org/0000-0002-2086-0679. nah.casarin@gmail.com

${ }^{3}$ Fisioterapeuta pela Universidade Federal de Santa Maria (UFSM). Mestrando em Reabilitação Funcional pela UFSM. http://lattes.cnpq.br/7962214089072791. https://orcid.org/0000-0001-9213-5209. maatheusbmoreira@gmail.com

${ }^{4}$ Fisioterapeuta pela Universidade Federal de Santa Maria (UFSM). Mestrado em Ciências da Saúde pela UFSM. http://lattes.cnpq.br/7192245416204928. https://orcid.org/0000-0001-9252-2057. gabrielagck@gmail.com

Fisioterapeuta pela Universidade Federal de Santa Maria (UFSM). Mestrado em Reabilitação Funcional pela UFSM. http://lattes.cnpq.br/2445089993660807. https://orcid.org/0000-0003-1989-4828. taitolves@hotmail.com

${ }^{6}$ Doutorado em Ciências da Saúde: Cardiologia, pelo Instituto de Cardiologia do RS - Fundação Universitária de Cardiologia (IC/FUC). Docente do Departamento de Fisioterapia e Reabilitação da Universidade Federal de Santa Maria (UFSM). http://lattes.cnpq.br/8302481057315339. https://orcid.org/0000-00017784-9940. I.signori@hotmail.com

Doutorado em Ciências Biológicas - Fisiologia pela Universidade Federal do Rio Grande do Sul (UFRGS). Docente do Departamento de Fisioterapia e Reabilitação da Universidade Federal de Santa Maria (UFSM). http://lattes.cnpq.br/9981854873337898. https://orcid.org/0000-0001-8127-6911. antonio.77@terra.com.br
} 


\section{INTRODUÇÃO}

A epidemia da infecção pelo Human Immunodeficiency Virus (HIV) e da Acquired Immunodeficiency Syndrome (Aids) representa fenômeno global, dinâmico e instável, cuja forma de ocorrência nas diferentes regiões do mundo depende, entre outros determinantes, do comportamento humano individual e coletivo (BRITO; CASTILHO; SZWARCWALD, 2000; CARPIO-CANO; CADENA; SAWAYA, 2013). Em virtude dos avanços da Terapia Antirretroviral (Tarv) e expansão do acesso ao tratamento, as mortes relacionadas à Aids caíram $30 \%$ desde o pico alcançado em 2005 , por sua principal função de retardar a progressão viral, além de ser a principal estratégia de tratamento para o HIV (MBADA et al., 2013). Embora seja responsável pelo controle virológico, um estudo recente sugere que ainda há algumas chances de falhas virológicas e imunológicas em indivíduos em uso da Tarv por pelo menos 6 meses (OJHA; SHAKYA; DUMRE, 2016). A carga viral e a contagem de células T-CD4 são importantes ferramentas para monitoração e acompanhamento da progressão do HIV. Sabe-se que pacientes com cargas virais elevadas têm risco aumentado de progressão para doença sintomática em comparação com doentes com níveis baixos ou indetectáveis (CARROL; ADAMS, 2016).

Apesar do desenvolvimento do tratamento ao paciente com HIV, ainda há evidências de diminuição da capacidade funcional (MBADA et al., 2013; JERÔNIMO et al., 2015), força muscular, qualidade de vida (PASSOS et al., 2012) e, consequentemente, deterioração da aptidão cardiorrespiratória (RASO et al., 2013). Relatos têm demonstrado função anormal das vias aéreas em indivíduos com HIV/Aids, como diminuição dos valores de pressões respiratórias máximas e de valores espirométricos (O'DONNELL et al., 1998; PASSOS et al., 2012), bem como a associação destas variáveis com o declínio na capacidade funcional (JERÔNIMO et al., 2015).

As alterações de força muscular respiratória podem ser explicadas pela perda de força muscular esquelética, que culmina em diminuição da capacidade ao exercício (OURSLER et al., 2009). Alterações bioquímicas foram correlacionadas com a Tarv (ARNAUDO et al., 1991) e com o vírus HIV (DE SIMONE et al., 1992), podendo sugerir disfunções mitocondriais nesta população. Isso culminaria em diminuição de suplementação de energia necessária para contração da musculatura esquelética e, consequentemente, musculatura respiratória (JERÔNIMO et al., 2015). Também foram encontradas associações entre função pulmonar e risco cardiovascular em pacientes com HIV (PASSOS et al., 2012), mas até o momento não se teve relatos do envolvimento da força muscular respiratória com doença cardiovascular.

A fraqueza muscular respiratória já foi apontada em estudos anteriores e está presente nos pacientes com HIV (PASSOS et al., 2012; RASO et al., 2013; JERÔNIMO et al., 2015), porém ainda não há relatos comparando diferentes níveis de carga viral. Considerando a crescente eficácia da Tarv na busca do adequado controle virológico, aliado aos seus efeitos sobre a musculatura respiratória e prognóstico cardiovascular, este estudo objetivou analisar a influência da Tarv e da carga viral sobre a força muscular respiratória e risco cardiovascular em pacientes com HIV.

\section{MATERIAIS E MÉTODOS}

Este estudo transversal foi aprovado pelo Comitê de Ética em Pesquisa da Universidade Federal de Santa Maria (UFSM), sob o protocolo número 30302414.0.0000.5346, conforme a Resolução 466/2012 do Conselho Nacional de Saúde. Todos os sujeitos assinaram o Termo de Consentimento Livre e Esclarecido.

A amostra por conveniência compreendeu 60 sujeitos portadores do vírus HIV, acompanhados no Ambulatório de Doenças Infecciosas do Hospital Universitário de Santa Maria e na Casa Treze de Maio, entre agosto de 2014 e maio de 2017. Foram incluídos sujeitos com HIV/Aids em uso de Tarv há pelo menos três meses ou sem uso, com carga viral não detectável ( $<50$ cópias $/ \mathrm{mL}$ ) e detectável ( $>50$ cópias $/ \mathrm{mL}$ ). Foram excluídos sujeitos com disfunção cognitiva que inviabilizasse as avaliações, com insuficiência cardíaca congestiva, marcapasso cardíaco, diabetes mellitus, doença de chagas, tuberculose, dependentes de álcool, em uso de drogas antiarrítmicas, betabloqueadores e estatinas, com doença vascular periférica, doença pulmonar obstrutiva crônica, neuropatias periféricas, doença cerebrovascular e/ou musculoesqueléticas crônicas e gestantes. Os pacientes foram divididos em três grupos: GTCV- (em uso da Tarv e carga viral indetectável), GTCV+ (em uso da Tarv e carga viral detectável) e GsTCV+ (sem Tarv e carga viral detectável).

Conforme a agenda dos locais de coleta, os sujeitos foram contatados logo após a consulta médica de rotina e convidados a participar. Mediante a entrevista e a revisão dos prontuários, foram registrados os dados para triagem conforme os critérios de exclusão. No dia da avaliação foram coletados os dados demográficos, antropométricos e sinais vitais. As informações clínicas, como o tempo de diagnóstico, o tempo 
de uso da Tarv, o valor da última contagem de células T-CD4 e carga viral, os níveis séricos de colesterol HDL e de colesterol total e o esquema de terapia antirretroviral, foram coletados do prontuário.

A força muscular respiratória foi estimada pela medida das pressões respiratórias máximas mensuradas com um manovacuômetro digital (MVD 300, Microhard System, Globalmed, Porto Alegre, Brasil) por um avaliador cegado, com o sujeito na posição sentada, utilizando um clipe nasal. Os valores previstos para pressão inspiratória máxima (PImáx) e pressão expiratória máxima (PEmáx) foram baseados nas equações propostas por Neder et al. (1999). Considerou-se fraqueza muscular inspiratória valores de PImáx < 70\% do predito (DALL'AGO et al., 2006). Valores de PEmáx $<70 \%$ do predito também foram levados em conta para definir fraqueza muscular expiratória.

Os sujeitos foram avaliados pelo escore de risco cardiovascular de Framingham, caracterizado por uma série de equações de predição desenvolvida a partir do estudo longitudinal Framingham Heart Study (BITTON; GAZIANO, 2010).
As análises estatísticas ocorreram pelo software GraphPad Prisma versão 5.0. Os dados foram apresentados em média e desvio padrão (DP), mediana e percentis (P25-75) e porcentagens. A distribuição dos dados foi avaliada pelo teste de normalidade de Kolmogorov-Smirnov. As variáveis categóricas foram avaliadas pelo teste de qui-quadrado. Para comparação de dois grupos foi utilizado o teste de Mann Whitney (entre o GTCV+ e GsTCV+). Os dados simétricos foram tratados com Anova de uma via e os assimétricos com o teste de Kruskal-Wallis, seguidos de post hoc de Tukey. Foi considerado o nível de significância de 5\% $(p<0,05)$.

\section{RESULTADOS}

Os grupos não diferiram quanto ao sexo, idade, índice de massa corporal, sinais vitais, tempo de medicação e Escore de Framingham (Tabela 1). O tempo de diagnóstico foi menor no GsTCV+ do que nos GTCV-e GTCV+. A contagem de T-CD4 foi menor no GTCV+ do que no GTCV-. A carga viral foi maior no GsTCV+ do que GTCV+.

Tabela 1 - Caracterização da amostra

\begin{tabular}{|c|c|c|c|c|}
\hline Variáveis & $\begin{array}{l}\text { GTCV- } \\
(n=20)\end{array}$ & $\begin{array}{l}\text { GTCV+ } \\
(n=20)\end{array}$ & $\begin{array}{c}\text { GsTCV+ } \\
(n=20)\end{array}$ & Valor de $p$ \\
\hline Sexo feminino $(n)$ & 12 & 10 & 9 & 0,626 \\
\hline Idade (anos) & $37,3 \pm 9,1$ & $35,5 \pm 9,9$ & $35 \pm 11,4$ & 0,745 \\
\hline $\mathrm{IMC}\left(\mathrm{kg} / \mathrm{m}^{2}\right)$ & $26,1 \pm 4,4$ & $25,6 \pm 5,9$ & $25,2 \pm 4,1$ & 0,825 \\
\hline $\mathrm{FC}(\mathrm{bpm})$ & $71,4 \pm 11,9$ & $75,5 \pm 9,5$ & $79,7 \pm 14$ & 0,098 \\
\hline $\mathrm{FR}(\mathrm{rpm})$ & $16,5 \pm 3,7$ & $17,8 \pm 5,2$ & $16 \pm 4,1$ & 0,399 \\
\hline PA Sistólica (mmHg) & $121,8 \pm 9,6$ & $124,5 \pm 20,1$ & $131,5 \pm 14,5$ & 0,127 \\
\hline PA Diastólica (mmHg) & $79 \pm 9,7$ & $76,3 \pm 18,1$ & $82,8 \pm 9,1$ & 0,290 \\
\hline Tempo de diagnóstico (meses) & $104,7 \pm 69$ & $103,7 \pm 72,7$ & $25,5 \pm 55,1^{*} \#$ & $<0,001$ \\
\hline Tempo de Tarv (meses) & $64,2 \pm 43,3$ & $61,8 \pm 61,0$ & Sem TARV & 0,456 \\
\hline Carga Viral (cópias/ml) & ND & $891,5(119,3-3527,5)$ & $81739(23937,8-142285,8)$ & $<0,001$ \\
\hline T-CD4 (céls/mm³) & $700,1 \pm 243,3$ & $498,8 \pm 289,6^{*}$ & $526,8 \pm 343$ & 0,042 \\
\hline Escore de Framingham (pontos) & $6,3 \pm 3,9$ & $5,6 \pm 4,8$ & $5,9 \pm 4,6$ & 0,682 \\
\hline $\begin{array}{l}\text { Escore de Framingham (\% de } \\
\text { risco) }\end{array}$ & $5,3(2,2-6,9)$ & $2,7(2,2-5,9)$ & $2,8(2,4-5,3)$ & 0,734 \\
\hline
\end{tabular}

Valores expressos em n, média \pm DP ou mediana (P25-75); IMC: índice de massa corporal; FC: frequência cardíaca, FR: frequência respiratória; PA: pressão arterial; TARV: terapia antirretroviral; ND: não detectável. *: diferença versus GTCV-; \#: diferença versus GTCV+. 
Quanto ao risco cardiovascular, os sujeitos do GTCV- foram classificados em baixo risco em $45 \%$ e risco intermediário em $55 \%$. No GTCV+, $70 \%$ dos sujeitos foram classificados como baixo risco, $20 \%$ risco intermediário e $10 \%$ alto risco. No GsTCV+ a maioria dos avaliados (70\%) apresentaram baixo risco, $25 \%$ risco intermediário e apenas $5 \%$ alto risco.

Em relação à Tarv, $55 \%$ dos sujeitos do GTCVutilizaram a classe de antirretrovirais Inibidores da Transcriptase Reversa Análogos de Nucleotídeos (INTRs) + Inibidores da Transcriptase Reversa Não análogos de Nucleosídeos (INNTRs), 40\% INTRs + Inibidores da Protease e $5 \%$ usaram INTRs. Já no GTCV+, $45 \%$ fizeram uso de INTRs + INNTRs, $40 \%$ INTRs + inibidores da Protease, $10 \%$ INTRs + INNTRs + Inibidores da Protease, 5\% INTRs + INNTRs + inibidores da Protease + Inibidores da Integrase.

A Tabela 2 demonstra que o GTCV+ e o GsTCV+ apresentam redução na PEmáx de $39,7 \%$ e de $32,3 \%$, respectivamente, na comparação com o GTCV-, baseado no \% do predito. Tanto o GTCV+ quanto o GsTCV+ apresentaram valores de mediana que demonstraram fraqueza muscular inspiratória, porém sem diferença significativa na PImáx comparado ao GTCV-.

\section{DISCUSSÃO}

Nossos achados mostram que pacientes com carga viral detectável, mesmo em uso da Tarv, apresentam fraqueza da musculatura expiratória na comparação com pacientes em uso da Tarv e carga viral indetectável. Também demonstramos que a maioria dos pacientes com carga viral detectável, independente da Tarv, apresenta fraqueza muscular inspiratória. O risco cardiovascular não identificou diferenças entre os três grupos, o que pode indicar que as alterações de força muscular respiratória foram independentes desta variável.
A hipótese de que os pacientes com HIV pudessem apresentar diminuição de força muscular respiratória começou com Schulz et al. (1997), quando observaram que sujeitos com HIV apresentaram redução da PImáx e PEmáx comparados a sujeitos saudáveis. 0 presente estudo não comparou estes pacientes com indivíduos saudáveis, porém avaliou pacientes que, teoricamente, têm uma vida saudável em comparação aos que estão expostos a infecções oportunistas (grupos com carga viral detectável). O controle da doença com a Tarv, neste estudo, é benéfico, pois o grupo que faz uso regular apresenta melhor valor de PEmáx e não traz fraqueza muscular inspiratória. Suspeita-se que os pacientes do GTCV+ não utilizavam Tarv de forma adequada, pois estes mantiveram-se com carga viral detectável. Recentemente, outro estudo analisou separadamente pacientes com HIV com e sem fraqueza muscular inspiratória, observando que o tempo de Tarv e contagem de células T-CD4 foram apontados como determinantes para fraqueza muscular inspiratória. Além disso, o grupo com fraqueza muscular inspiratória e contagem de células T-CD4<200 apresentou predominância de pacientes com carga viral detectável (JERÔNIMO et al., 2015). O controle virológico parece ser benéfico para evitar a fraqueza muscular inspiratória, pois, em ambos os estudos, a carga viral detectável esteve presente nos grupos com fraqueza.

A maior causa de mortalidade e morbidade em pacientes com HIV são as complicações pulmonares (PALELLA et al., 1998). Depois da Tarv, houve uma diminuição da incidência de doenças oportunistas, como infecções pulmonares (MOORE; CHAISSON, 1999). Antes dela, porém, alguns estudos investigaram pacientes com HIV e relataram uma obstrução acelerada das vias aéreas, bem como sintomas respiratórios mais frequentes quando comparados com um grupo controle (DIAZ; CLANTON; PACHT, 1992;

Tabela 2 - Valores das pressões respiratórias obtidas e percentuais do predito

\begin{tabular}{|c|c|c|c|c|}
\hline & $\begin{array}{l}\text { GTCV- } \\
(n=20)\end{array}$ & $\begin{array}{l}\text { GTCV+ } \\
(n=20)\end{array}$ & $\begin{array}{c}\text { GsTCV+ } \\
(n=20)\end{array}$ & Valor de $p$ \\
\hline PImáx $\left(\mathrm{cmH}_{2} \mathrm{O}\right)$ & $79,5(59-98,5)$ & $70,5(46-88)$ & $77,5(54,8-99)$ & 0,495 \\
\hline PImáx (\%pred) & $72,6(59,3-96)$ & $63,2(44,3-77,4)$ & $65,1(48,6-89)$ & 0,185 \\
\hline FMI (\%) & 50 & 60 & 55 & \\
\hline PEmáx $\left(\mathrm{cmH}_{2} \mathrm{O}\right)$ & $102,5(80-129,3)$ & $61,5(40,8-91,8)$ & $91(68,8-118,5)$ & 0,063 \\
\hline PEmáx (\%pred) & $100,2(71-121,9)$ & $60,5(37,1-70,5)^{*}$ & $67,9(50,3-93)^{*}$ & 0,004 \\
\hline FME (\%) & 20 & $75^{*}$ & $50 *$ & \\
\hline
\end{tabular}

Valores expressos em mediana (P25-75). PImáx: pressão inspiratória máxima; FMI: fraqueza muscular inspiratória; PEmáx: pressão expiratória máxima. \%pred: percentual do predito; FME: fraqueza muscular expiratória.

*: diferença versus GTCV- 
WALLACE et al., 1997; GINGO et al., 2010). Embora não tenhamos comparado nossos achados com um grupo de indivíduos saudáveis, os resultados encontrados pelas pesquisas anteriormente citadas podem explicar os resultados negativos do presente estudo, posto que a função pulmonar e a força muscular respiratória já foram associadas nesta população (JERÔNIMO et al., 2015).

A presença de fraqueza muscular inspiratória nos pacientes que usam Tarv, porém, com carga viral detectável, destaca a importância de adesão ao tratamento. Estudos anteriores apontam a associação positiva entre qualidade de vida desta população e sua adesão ao tratamento (MANNHEIMER et al., 2005; PARSONS et al., 2006). O aumento da sobrevida pela evolução do estado crônico da doença, no entanto, pode não implicar melhoria da qualidade de vida global, pois a maior adesão pode aumentar os efeitos colaterais das medicações (GROCZE et al., 2010).

$O$ escore de Framingham tem sido utilizado nessa população a fim de avaliar a probabilidade de evento cardiovascular em razão dos efeitos potencializadores da infecção pelo HIV e do uso da terapia medicamentosa (ASZTALOS et al., 2014; ZHOU et al., 2015). Nossos achados demonstraram prevalência de baixo risco de evento cardiovascular, com similaridade entre os grupos. Isso sugere que as diferenças na carga viral e contagem de T-CD4 não foram capazes de determinar o risco cardiovascular, o que pode ser explicado, pelo menos em parte, pela reduzida faixa etária e pela similaridade na idade, sexo e índice de massa corporal entre os grupos. Ainda, a exclusão de pacientes com diagnóstico de diversas comorbidades nos levou a avaliar uma amostra com menor comprometimento de saúde e melhor estabilidade clínica, o que pode ter atenuado a observação de maior risco cardiovascular. Este achado é reforçado por alguns estudos recentes, relatando que $97 \%$ dos pacientes com HIV apresentaram baixo risco de doença cardiovascular, também avaliado pelo Escore de risco de Framingham (ZHOU et al., 2015) e que o uso precoce da Tarv não foi correlacionado com o aumento do risco cardiovascular em 2.056 pacientes com HIV, acompanhados durante 30 meses (GHEHI et al., 2017). Mesmo em pacientes soropositivos e com baixo risco cardiovascular, no entanto, tem sido relatada uma alta prevalência de aterosclerose carotídea, sendo a idade e o perfil inflamatório os principais fatores de risco (LEÓN et al., 2017).

A diferença entre os tempos de diagnóstico deve-se ao fato de que os pacientes que não fazem o uso da Tarv neste estudo são os que possuem diagnósti- co recente. Os pacientes do GsTCV+ foram abordados para coleta na sua primeira consulta médica, logo após a confirmação do diagnóstico, e por isso estavam sem prescrição de Tarv. Esta ausência de prescrição de Tarv neste grupo parece não ter influenciado os nossos achados de força muscular respiratória, uma vez que o grupo com Tarv e carga viral detectável também apresentou redução similar da PEmáx. Com o advento da Tarv, é difícil encontrar pacientes em ambulatórios de infectologia que apresente diagnóstico recente e tempo prolongado de HIV sem tratamento. Nossos resultados podem sugerir que o vírus já tem uma ação evidente mesmo em indivíduos assintomáticos (BRASIL, 2013).

Nosso estudo apresenta limitações, como pequena amostra e ausência de um grupo controle. Os valores previstos das pressões respiratórias máximas foram determinadas por intermédio de Neder et al. (1999), no entanto, por ser um estudo que começou a ser desenvolvido em 2014, infelizmente não foi possível a utilização da fórmula mais atual proposta por Pessoa et al. (2014) pela falta da medida de cirtometria abdominal.

\section{CONCLUSÃO}

O grupo de pacientes em uso de Tarv e com carga viral não detectável apresentou maior força muscular expiratória e não foi classificado com fraqueza muscular inspiratória. Os resultados apontam para o efeito benéfico do adequado controle virológico e para a importância da adesão precoce ao tratamento. Nossos achados sugerem que a musculatura respiratória, especialmente os músculos expiratórios, podem mostrar-se como um importante alvo terapêutico nesta população. Estes efeitos favoráveis da Tarv e da baixa carga viral podem implicar melhores desfechos funcionais. Assim, programas de reabilitação voltados à melhora da força muscular respiratória em pacientes com HIV, merecem ser testados em futuras investigações.

\section{REFERÊNCIAS}

ARNAUDO, E. et al. Depletion of muscle mitochondrial DNA in Aids patients with zidovudine-induced myopathy. Lancet, v. 337, n. 8.740, p. 508-510, 1991.

ASZTALOS, B. F. et al. Cardiovascular disease-risk markers in HIV patients. Journal of AIDS \& Clinical Research, v. 5, n. 7, p. 1-21, 2014.

BITTON, A.; GAZIANO, T. The Framingham Heart Study's Impact on Global Risk Assessment. Progress in Cardiovascular Diseases, v. 53, n. 1, p. 68-78, 2010. 
BRASIL. Ministério da Saúde. Secretaria de Vigilância em Saúde. Departamento de Vigilância, Prevenção e Controle das Infecções Sexualmente Transmissíveis do HIV/Aids e das Hepatites Virais. Protocolo Clínico e Diretrizes Terapêuticas Para Manejo da Infecção pelo HIV em Adultos. Brasil: MS, 2013.

BRITO, A. M.; CASTILHO, E. A.; SZWARCWALD, C. L. Aids e infecção pelo HIV no Brasil: uma epidemia multifacetada. Revista da Sociedade Brasileira de Medicina Tropical, v. 34, n. 2, p. 207-217, 2000.

CARPIO-CANO, F. E. D.; CADENA, R. A. D.; SAWAYA, B. E. HIV and Bone Disease: A Perspective of the Role of microRNAs in Bone Biology upon HIV Infection. Journal of Osteoporosis, v. 2.013, n. 571.418, p. 1-8, 2013.

CARROL, K. C.; ADAMS, L. L. Lower Respiratory Tract Infections. Microbiology Spectrum, v. 4, n. 4, p. 539-540, 2016.

DALL'AGO, P. et al. Inspiratory muscle training in patients with heart failure and inspiratory muscle weakness: a randomized trial. Journal of the American College of Cardiology, v. 47, n. 4, p. 757-763, 2006.

DE SIMONE, C. et al. L-carnitine deficiency in Aids patients. Aids, v. 6, n. 2, p. 203-205, 1992.

DIAZ, P. T.; CLANTON, T. L.; PACHT, E. R. Emphysema-like pulmonary disease associated with human immunodeficiency virus infection. Annals of Internal Medicine, v. 116, n. 2, p. 124-128, 1992.

GHEHI, C. et al. High correlation between Framingham equations with $\mathrm{BMI}$ and with lipids to estimate cardiovascular risks score at baseline in HIV-infected adults in the Temprano trial, ANRS 12136 in Côte d'Ivoire. PLoS One, v. 12, n. 6, e0177440, 2017.

GINGO, M. R. et al. Pulmonary function abnormalities in HIV-infected patients during the current antiretroviral therapy era. American Journal of Respiratory and Critical Care Medicine, v. 182, n. 6, p. 790-796, 2010.

GROCZE, L. et al. Qualidade de vida e adesão ao tratamento anti-retroviral de pacientes portadores de HIV. Revista de Saúde Pública, v. 44, n. 4, p. 743-749, 2010.

JERÔNIMO, F. S. et al. Factors associated with inspiratory muscle weakness in patients with HIV-1. The Brazilian Journal of Infectious Diseases, v. 19, n. 1, p. 1-7, 2015.

LEÓN, R. et al. Subclinical atherosclerosis in low Framingham risk HIV patients. European Journal of Clinical Investigation, v. 47, n. 8, p. 591-599, 2017.

MANNHEIMER, S. B. et al. Quality of life in HIV-infected individuals receiving antiretroviral therapy is related to adherence. Aids Care, v. 17, n. 1, p. 10-22, 2005.

MBADA, C. E. et al. Health-related quality of life and physical functioning in people living with HIV/Aids: a case-control design. Health and Quality of Life Outcomes, v. 106, n. 11, p. 1-8, 2013.

MOORE, R. D.; CHAISSON, R. E. Natural history of HIV infection in the era of combination antiretroviral therapy. Aids, v. 13, n. 14, p. 1.933-1.942, 1999.
NEDER, J. A. et al. Reference values for lung function tests. II Maximal respiratory pressures and voluntary ventilation. Brazilian Journal of Medical and Biological Research, v. 32, n. 6, p. 719-727, 1999.

O'DONNELL, C. R. et al. Abnormal airway function in individuals with the acquired immunodeficiency syndrome. Chest, v. 94, n. 5, p. 945-948, 1998.

OJHA, C. R.; SHAKYA, G.; DUMRE, S. P. Virological and Immunological Status of the People Living with HIV/Aids Undergoing ART Treatment in Nepal. BioMed Research International, v. 2.016, n. 6.817.325, p. 1-7, 2016.

OURSLER, K. K. et al. Prediction of cardiorespiratory fitness in older men infected with the human immunodeficiency virus: clinical factors and value of the six-minute walk distance. Journal of the American Geriatrics Society, v. 57, n. 11, p. 2.055-2.061, 2009.

PALELLA, F. J. et al. Declining morbidity and mortality among patients with advanced human immunodeficiency virus infection. HIV Outpatient Study Investigators. New England Journal of Medicine, v. 338, n. 13, p. 853-860, 1998.

PARSONS, T. D. et al. Better quality of life with neuropsychological improvement on Haart. Health and Quality of Life Outcomes, v. 24, n. 4, p. 11, 2006.

PASSOS, A. I. M. et al. Evaluation of Functional Respiratory Parameters in Aids Patients Assisted in the Infectious Diseases Ambulatory Care Clinic of a Tertiary Care University Hospital in Brazil. Respiratory Care, v. 57, n. 4, p. 544-549, 2012.

PESSOA, I. M. et al. Predictive equations for respiratory muscle strength according to international and Brazilian guidelines. Brazilian Journal of Physical Therapy, v. 18, p. 2, p. 410-414, 2014.

RASO, V. et al. Association between muscle strength and the cardiopulmonary status of individuals living with HIV/ Aids. Clinics, v. 68, n. 3, p. 359-364, 2013.

SCHULZ, L. et al. Respiratory Muscle Dysfunction Associated with Human Immunodeficiency Virus Infection. American Journal of Respiratory and Critical Care Medicine, v. 155, n. 3, p. 1.080-1.084, 1997.

WALLACE, J. M. et al. Respiratory disease trends in the pulmonary complications of HIV infection study cohort. Pulmonary Complications of HIV Infection Study Group. American Journal of Respiratory and Critical Care Medicine, v. 155, n. 1, p. 72-80, 1997.

ZHOU, D. T. et al. Dyslipidemia and cardiovascular disease risk profiles of patients attending an HIV treatment clinic in Harare, Zimbabwe. HIV/Aids - Research and Palliative Care, n. 7, p. 145-155, 2015. 